\title{
A Study of Poetry Design from the Perspective of Cognitive Development
}

\author{
Shu Hsuan Chang \\ National Taiwan University of Arts, Taiwan
}

\begin{abstract}
This study develops an assessment (estimating) mode by exploring the theories of poetry and design. Then, it tests the assessment (estimating) mode from product perceptions and poetry conceptions. According to the six observing principles from the Chinese literary theory, wen xin dio long, the questionnaire survey would examine the product perceptions, color preferences and poetry conceptions, making inferences for the estimating method between product perceptions and poetry conceptions specifically. Moreover, it analyzes how to improve the product images, match the poetry conceptions, and measure the difficult levels of assessment indicators (estimating indexes) when the poetry meanings were transferred. Therefore, product design could communicate traditional poetry culture concretely. It is difficult to take a scientific approach exactly to interpret artistic conceptions. During this study, setting the assessment indicators (estimating indexes) and categorizing the poetry meanings might be affected by some subjective reasons. However, it still promotes the reproductions through literature to art obviously. In summary, the findings of assessment (estimating results) between poetry meanings and product perceptions could support the externals of product design. The inheritance and inspiration of poetry culturally creative design can be effectively extended.
\end{abstract}

Keywords: poetry, creative design, cultural creative

\section{Preface}

It is a prevailing era that the world is entering a culturally creative industries and aesthetic economy. Lin, R. T. (2010) pointed out that "culture" is a lifestyle; "design" is a taste of life. "Creativity" is a kind of recognition touched by heart, and "industry" is medium, means or methods which make the cultural and creative design possible. In the culture aspect, the design, through culturally creative industries, makes a design taste possible in a form of a lifestyle; that is the best interpretation of "initial in the culture, shaped with creativity, applied to the daily life”. Chinese traditional "poetry” features creative design applications with a cultural connotation. It will enhance a unique identification for a product, and give a design with a newly aesthetic implication, becoming a mode of important culturally creative thinking in an international cross-cultural sector.

Wang, M.O. (1976 ) once said that a writer would make an aesthetic metaphor in his/her imagery of emotions, expressing in language, or other symbols. However, a reader would stand in an opposite perspective, his/her imagery of emotions from the language or symbols are restored. The readers also enjoy an aesthetic

Shu Hsuan Chang, Ph. D. Candidate, National Taiwan University of Arts.

Correpondence concerning this article should be addressed to No.2, Ln. 41, Zhengguang St., Taoyuan Dist., Taoyuan City 330, Taiwan (R.O.C.). E-mail: mikejsc@gmail.com. 
experience in the process of such an restoration. It therefore can explain the extreme values of literature closely relating to aesthetic experiences and beautiful feelings. Tai ,C.H. (2009) pointed out that a perception process is a reversing work of brains. Using experiments and simulations and other methods to understand the brain's cognitive processes (algorithms) and characterization (data structures) can not only guide observations but also interpret the observed evidence.

Moreover, the thoughtfully specific modes for creative design in a poetry culture would echo the above statement, becoming an excellent annotation for the transformed poetry. By means of the "culturally creative design application on poetry transformation", the "culturally creative design applications on temporal form of poetry transformation” and other series of studies and further explorations of related applications on poetry culture design. In an attempt to seek the empiricism theoretical concepts by David Hugh, and in a selection of six observing principles of "wen xin dio long" as assessing indicators. Poetry elements of a poet from deconstructing to restructuring are in the cognitive process for culturally creative product design. An assessing method for concrete operations is to be sought to.

Clarify the relational construct formed between these two cognitive concepts and feasibility of concrete operations. Although poetry screening or analysis is influenced by some subjective factors, such as viewpoints, personal learning and knowledge, and the level of understanding, the British scholar Coleridge expressed Yu, C.Y. (2006) that "artistic creation theory" proposed a structured proposition, the "equal-view theory of translucent compartment”. Most great works were described in the use of this kind of structure: it allows general recipients to enjoy a basic surface; meanwhile, high-level recipients may have a deep glimpse; moreover, some transitional recipients may have doubts and wander between these two levels before they enter the inquired beautiful life, which happens to hold the same view with the creative design applications on poetry culture.

\section{Literature}

This chapter will deal the thinking mode of creative design on poetry culture. Hume's theory on impression ideas and the assessment concepts of this study is designed to set up six observing principles as assessing indicators, and emotional design applications and other related topics are used in exploration as below.

\section{Creative Design Thinking of "Poetry Culture"}

Li, Y.Y. (1996) argued that culture was referred to "a product produced by the human being, a law system sustaining a social life, an art product for a spiritual life; meanwhile, it also includes many human mental activities in the creation process". It was inherited five thousand years of Chinese history and classical "poetry" culture was handed down from generations to generations. Undoubtedly, such a poetry culture is an intangible cultural asset and a most important and valuable part. As the German philosopher Cassirer (1944) puts it, culture is kind of externalization, an object-oriented one; it is also a symbol of the reality and specific form in activities.

Symbolic form is in the culture form of all human. American philosopher Langer (1953) was also deeply affected by the advocates of "Art is the creation of forms symbolic of human feeling”, becoming an influential aesthetic theory in modern history. Nevertheless, the poetry culture is exactly a form of figurative-text symbols, an artistic performance to express psychological emotions. German philosopher Worringer (2007) in his book, 
entitled "Abstract and Empathy", pointed out that humans could get happiness from art, mainly because humans could take the individual things of outside world away from the vagaries of chance, and transform them into eternity in an abstract form. The occurrence of "poetry" is what Chu, K.C. (1988) said, "Depression in life came from the dissatisfaction of "being limited". Fantasy is to seek an "unlimited” life. It further noted: "poetry contains meaning internally and externally, internally it expresses reasons; externally it expresses images. Both they are internally and externally implicit so they may enter a poetry world”. In short, it is the language in which to pursue an unlimited space of imagination, and the image of the poem is built in the architecture of a logical thinking. Rapaille (2006) stated, “a good product must have an ability to activate consumers' cultural symbols"; under such a premise, we may have an expectation to decipher the "the poet's emotions" through the long-lived classical poetry culture, The design aims to be converted into culturally creative "poetic" products to comply with the demand and hunger for modern people's empty hearts. Furthermore, the meaning and depth of the culturally creative design can be thus enhanced.

\section{Evaluating Theoretical Concepts of "Impression Concept"}

In 18th century, a British philosopher David Hume (1711-1776) in his writing "Treatise of Human Nature" divided all human perceptual experiences into two categories. One is "impressions", a sensory perception, joined by the sensory impression and introspection impression, i.e. perception or memory; the other is " "ideas"; the idea is a copy of the impression, through the necessary links, it leads to new ideas, shown in "Figure 1". Among them, seven different philosophical concepts of the principles of the association of ideas are contained, such as resemblance, relations of time and place, and causation. From knowledge interpreted by Hume's experimentalism, the human can learn the correlation between human perception to memory and imagination processes in this research, such as impressions and ideas of "poetry sense and poetry conceptions". They come from the linkage of ideas generated by the scene in life. Such ideas are similar or close to the daily lives, our educational process, emotional experiences, etc. When we repeatedly see or experience poetry design concepts, in a created atmosphere, we would arouse a sentiment to urge us to reproduce poetry. Further, our memory would be strengthened to develop a richer imagination; that is, the eternal poetry has been reproduced with a modern design. This study thus uses Hume's "impression" and "idea" to be the basis of theories. And then Hume's "impression" idea would be set as "poetry product impression". After test, if the poetry color impression was reproduced in kind of "memory" type of "sensation", such an impression will be classified as the cognitive impression of "simile or analog" type. If "imagination" of "reflection" is the type, it will be classified as the cognitive impression of "metaphor or metonymy" type. Furthermore, Hume's "impression" idea would be set as "poetry conception and idea". With the help of assessment indicators covered within six observing principles, we can understand the meaning of poetry (Bixing themes), meaning of words (semantics), Palace Quotient (QingCai Rhyme), Body (layout structure), Fantasy (hyperbole innovation), and poetry flexibility (change through the times) and other types; assessment indicators are used to convert the degree of difficulty in performance.

\section{Design of "Six Observing Principles" to Convert into Implications}

"Wen xin dio long” aims to include the physical and metaphysical scope of formative form, activities , phenomenon, and functions of the nature of the universe, universal attributes and the world. The range can be converted from a macro perspective of philosophers, widespread literature and nature of the universe, the flowers in the world, social and political society, symbolic relationship between the meaning of life. In this 
study, "Wen xin dio long" proposed six observing" assessment indicators to build up the product analysis and poetry perceptions.

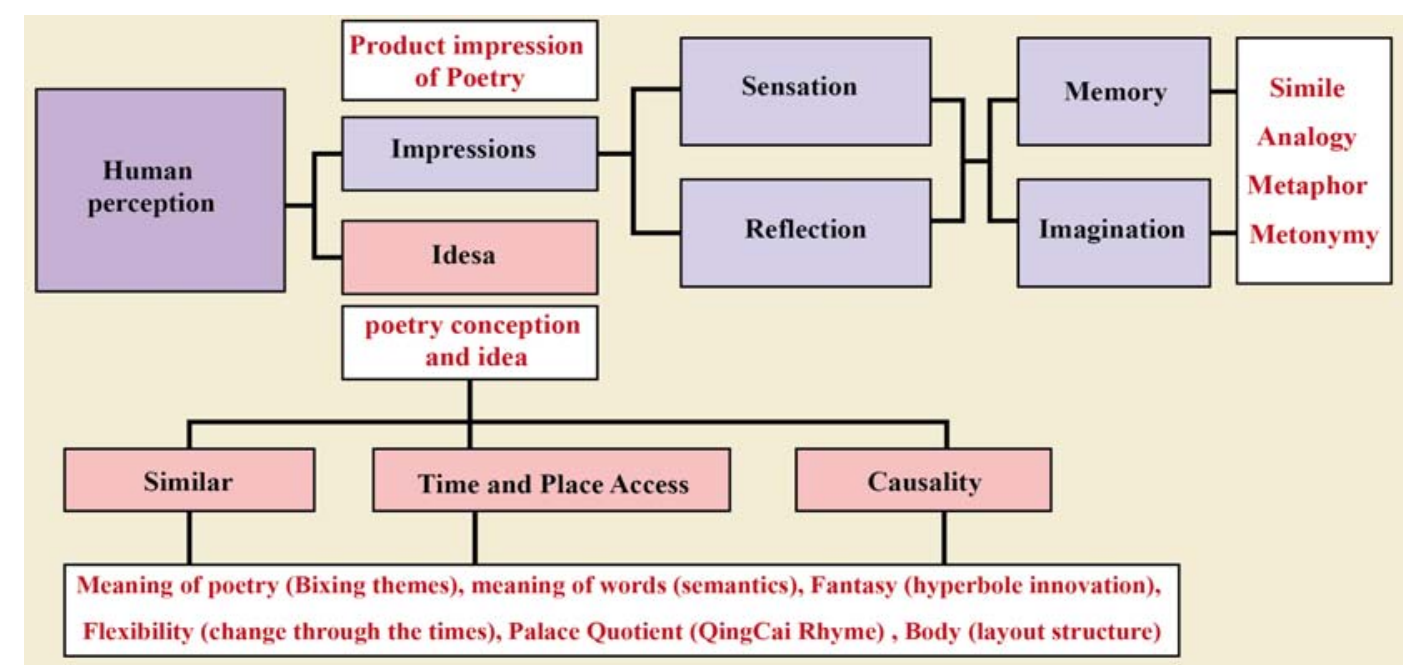

Figure 1. Correlation of Hume's experimentalism and this Study.

The so-called "When a person reads an article, he/she will first make six observations: one is to observe body; second meaning of words; third flexibility; forth fantasy; fifth meaning; sixth palace quotient”. Since such skills are practiced, the pros and cons will be noticed. In short, "body" (layout structure) is to see whether or not reasoning and stylistic configuration is appropriate, and the symbolic character should be shaped in the design elements; "meaning of words" (semantics) is to see whether or not the aesthetic norms of the words and phrases can be conformed in terms of the usage of diction; in the adaptability design, it can conform to the designed key attributes of the theme; "flexibility" (Change through the times) is to see how far the achievement can go in terms of style in convention inheritance and innovation progress, and it can conform to the designed adaptability principle in proportionality can be followed; "Fantasy" (hyperbole innovation) is to see whether or not the typical or cunning writing is appropriate in strategies, and should be conformed to the degree of meaning, emotions and innovation. "Meaning of poetry" (Meaning of Topic Material and Classical Citations) is to see whether or not the dispatch of material is well developed according to the situation; it can conform to the performance of the designed material texture and color. Palace Quotient (Syllables) is to see whether or not the operation of rhythm is naturally fluent in circumflex, and it can conform to the performance of designed dynamic situations and atmosphere. Therefore, this study uses six observing assessment indicators, which serve as the basis of poetry attribute transformation, also serve as the references of assessing the overall performance of whole works with its set standard.

In every dimension, the conversion design considerations and modes can be used according to the actual demands. Several dimensions in pairs can be applied or all dimensions can be integrated in application.

\section{Research and Framework}

\section{Structure and Process of Assessment Mode}

This study aimed to explore how to people recognize the related factors of the "ideas of poetry impression" and the influence over product designs. It is hoped that the assessment modes concretely operated between these two items can be sought. The research framework is shown in "Figure 2". 


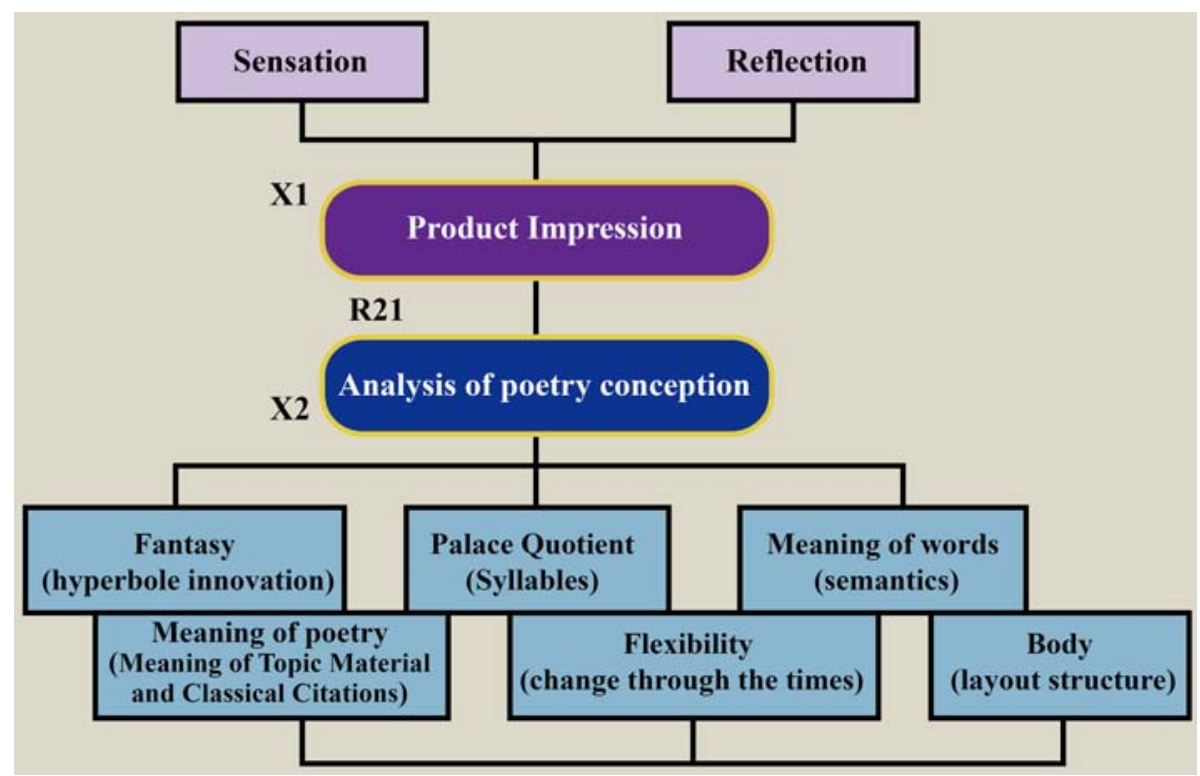

Figure 2. Research Framework of Assessment Mode

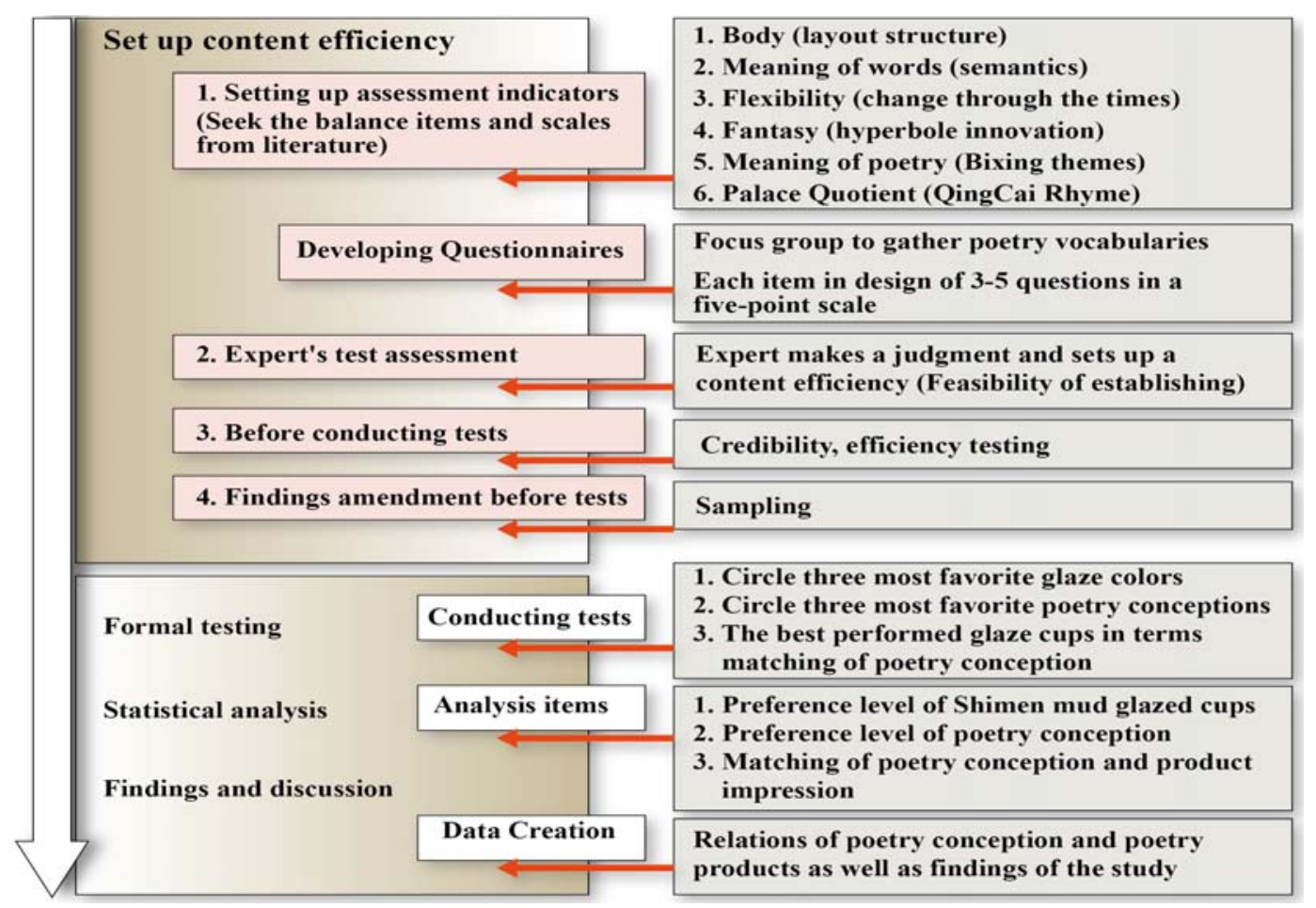

Figure 3. Development process of Assessment Mode.

Questionnaires are divided into two parts; the first part deals with the testees' basic background information, including sex and age, the second part deals with the test color preference, poetry artistic preference, product impression and poetry conception should be conformed to for the testing of three items. In "Figure 3" it shows the quantitative assessment of cognitive tests; the chosen test sample is mud from the Shimen Reservoir, adding all kinds of glazes on the mud to make glazed cup. Select six Shimen mud cups with more distinctive features and higher degree of differences. Its presentation of the artistic conception of tested 
poetry in pictures on the cup body in dense colors. People can fully experience and feel the artistic effect of poetry. In testing the color preference, partial color samples are represented in order to minimize the interference due to the shape. In scale design part, the focus group first collected poetry-related vocabulary and selected poetry, including the expression of colors and glazed cups and artistic poetry. It covered as much as possible "six observing principles". Each product had three samples corresponding to the poetry, including "simile, metonymy, and metaphor". Preliminary tests and screening were carried out by experts, and then poetry vocabularies were conformed; eventually six samples were compiled with three corresponding poems, eighteen poems in total. Then testing was conducted and statistical analysis were made on glaze preference, poetry artistic preference, poetry and color impression in adaptability, and poetry and artistic ideas in in adaptability. In a five-point scale, "extremely match" represents five points, and then in sequence to "extremely not match" represents one point. Finally, the statistical results showed that in the category of "impression" and "Idea", the practical understanding of the idea of poetry impression and product design would be recognized in terms of effect.

\section{Result and Discussion}

This chapter deals with the color preference (Test A) after test, the matching the degree of product perception and poetry conception (Test B), and poetry artistic preference (Test C). These three items were conducted with questionnaires, and then every single item was cross-compared in the preliminary quantitative analysis and qualitative analysis, and the correlation and findings of preliminary studies have been found, shown as "Figure 4" Finally, in views of product perception and poetry conception, we can generalize assessment methods with concrete operations, degree of difficulty of related matching adaptability, and six observing assessment indicators to convert the degree of difficulty of items.

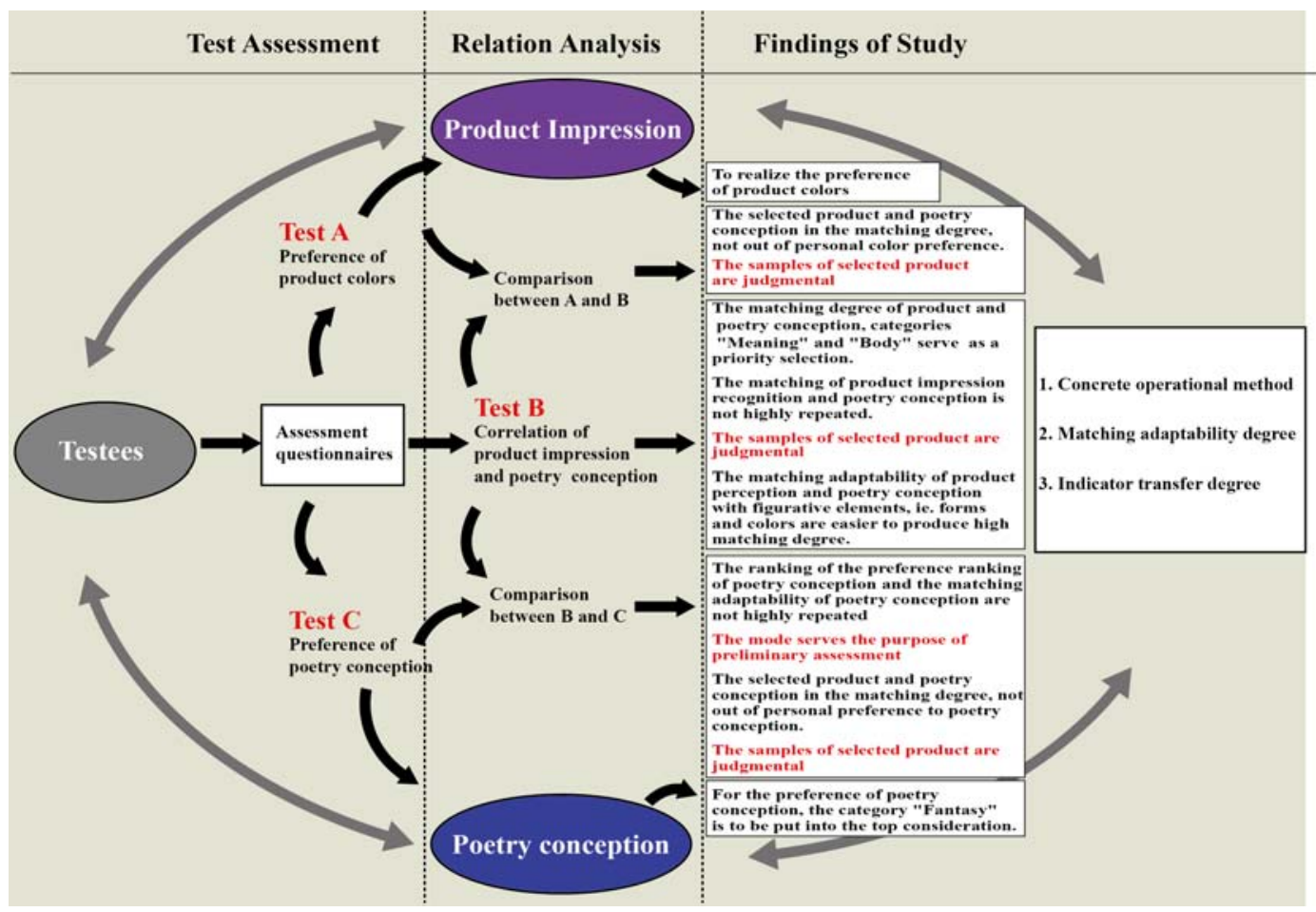

Figure 4. Relations Assessment Diagram of Product Impression V.S poetry conception. 


\section{Product Color Preference Analysis (Test A)}

Testees' Product (Shimen Cup) Color Preference Analysis, shown in “Table 1”.

Table 1

Color Preference Analysis of Shimen Mud Glazed Cup

\begin{tabular}{lllll}
\hline Sample & N & Mean & SD & Ranking \\
\hline a1 (power-greeen) & 27 & 1.89 & 1.121 & 1 \\
a2 (iron-red) & 27 & 0.78 & 1.050 & 2 \\
a3(Ugyen-gold) & 27 & 1.04 & 1.192 & 3 \\
a4(goose-yellow) & 27 & 0.96 & 1.192 & \\
a5(Syrup-glaze) & 27 & 0.44 & 0.801 & \\
a6(Leopard-spot) & 27 & 0.85 & 1.064 & \\
\hline
\end{tabular}

\section{Comparison Between Test A and Test B: Matching Adaptability of Product Perception and Poetry Conception and Comparative Analysis of Product Color Preference}

Matching adaptability of product color and poetry conception are independent of personal color preferences Where Table 2. In "Poetry conception preference", "When walking to a place where there is no water, Sit and watch clouds in the sky", which is the second sorting at the third ranking in the preference the "Goose-yellow glazed cup"; at the second ranking in poetry conception preference "Watch the bright moon through light clouds; see the green mountains through light fog”, which is the third sorting at the first ranking in the product preference the "powder-green glazed cup"; It showed that the testees' selection of product and poetry conception in the matching degree, not out of personal color preference. This study also shows the selected product samples are judgmental, for the poetry product design it is recommended that poetry implications should be consistent with the direction of theme.

Table 2

poetry conception Preference Analysis (Test B)

\begin{tabular}{llll}
\hline No & Poetry & Ranking \\
\hline No.13 & "When walking to a place where there is no water, Sit and watch clouds in the sky" & 3.15 & 1 \\
No.03 & "Watch the bright moon through light clouds; see the green mountains through light fog” & 2.81 & 2 \\
No.22 & "Every cloud has a silver lining” & 2.74 & 3 \\
No.23 & "But afraid of those large mansions; too high to keep out the cold” & 2.56 & 4 \\
No.01 & "Beautiful Views of Fresh Green Mountain Peaks after Rain" & 2.52 & 5 \\
\hline
\end{tabular}

\section{Matching Degree Analysis of Product Perception and Poetry Conception (Test B)}

To test the product perception and matching adaptability of poetry artistic conception, in this study, we selected 24 poems for subjects in views of various glazed cups, in the five-point scale assessment, to assess the matching adaptability of each poem and glazed color of the cup. In which, powder-green glaze is most suitable for the top-three poetry in ranking and their averages have been obtained with one-sample $t$ test. The findings are shown in Table 2. First place "Beautiful Views of Fresh Green Mountain Peaks after Rain"; second place "Green in Autumn Waters as White on Silver"; third place "Watch the Bright Moon through Light", whose averages are all greater than the hypothetical average No. 3. Goose-yellow glaze is the most suitable average number with "one-sample t test" of the top three poems. Among them, first place "Green in Autumn Waters as White on Silver" and second place "When walking to a place where there is no water, Sit and watch clouds in 
the sky", whose averages fail to reach a significant level; even though third place "Flowers in a mirror and the moon on water are elusive in an end", whose average is 3.33 same as that of second place, its standard difference is smaller yet $t$ value is greater than that of second place, so a significant level is reached $(p<.05)$.

Ugyen glaze is most suitable for the "one-sample t test" average of the top three poems, in which first place "New comb inserted into black thick hair", second place "Looking back, I saw that person was standing in dim lights" and third place "Chuang Sheng's daydream with butterflies”, whose averages fail to reach a significant level; Leopard droplet glaze is most suitable for the "one-sample t test" average of the top three poems, in which first place "ten-thousand stars falling on the earth", second place "Frost makes the forest withered, and yellow leaves are flying in all directions" and third place "Tears stream down and wet the gorgeous dress", whose average reaches a significant level. Iron-red crystalline glaze is most suitable for the "one-sample t test" average of the top three poems, in which first place "In sunrise flowers along the river are red like fire", second place "Tears stream down and wet the gorgeous dress" and third place "Fallen flowers are not heartless things, transforming into fertilizers in Spring for benefiting flowers", whose averages all reach a significant level. Syrup glaze is most suitable for the "one-sample t test" average of the top three poems, in which only the average of first place "Leaves are rustling and falling down" reaches a significant level; second place "Fallen flowers are not heartless things, transforming into fertilizers in Spring for benefiting flowers", and third place "In sunrise flowers along the river are red like fire", whose averages fail to reach a significant level.

Shimen Mud glaze cup and poetry conception matching analysis and one-sample $t$ test of the top three fitted poetry scoring.

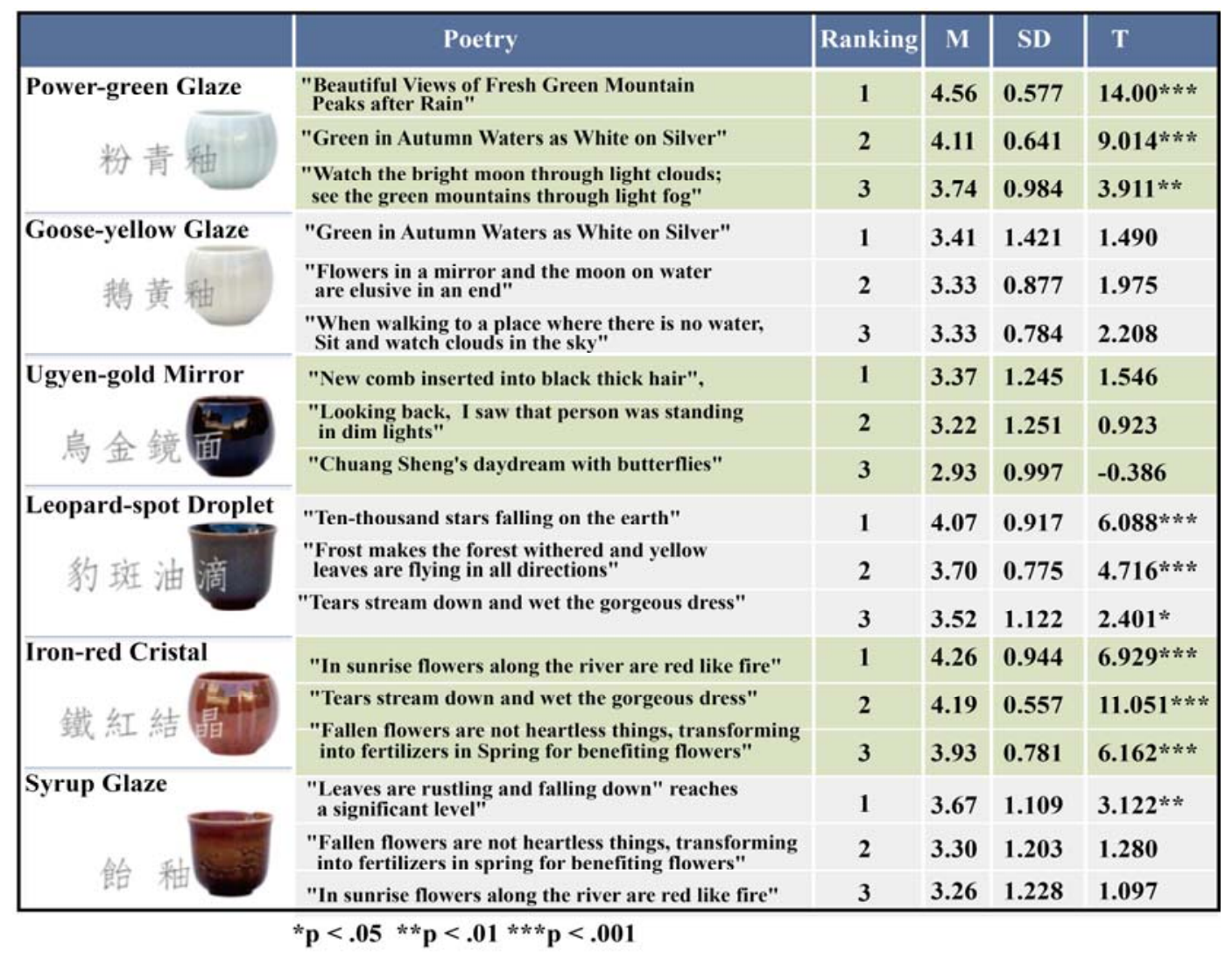


In terms of the matching adaptability of product perception and poetry conception, "the meaning of poetry", "the body of poetry" are regarded as a high matching level.

The analysis of "Matching level of product perception and poetry conception" in Table 3 showed that the meaning of poetry ranked the number 1 in the six observing principles. Followed up in sequence are "the analogies of colors" (4.3 3.1 3.2 1.1), "the metonymy of colors" (5.1 1.2 1.3 2.1)and "the metaphor of colors" ( 2.22 .2 at the same rank). The second ranking is the body of poetry (two cups out of six cups are top one), in which followed by the analogies of the body (6.3 6.2 6.1) and the metonymy of the body (4.1). In six observing principles, it showed that the meaning of poetry is the top one in terms of the easiest operation matching level (four cups out of six cups are top one).

The matching adaptability of product perception and poetry conception are not highly repeated

In Table 3, each glazed cups had been respectively checked by 24 poems, six glazed cups in total of 144 times. Among them, 18 poems in total were ranked for the top 3; There were only 3 poems listed, respectively ranking No. 1 "When walking to a place where there is no water, Sit and watch clouds in the sky"; ranking No. 2 "Watch the bright moon through light clouds; see the green mountains through light fog"; ranking No. 5 "Beautiful Views of Fresh Green Mountain Peaks after Rain”. It showed that the matching adaptability of product impression recognition and poetry conception (impression and idea) is not highly repeated. The selected product samples in this study are judgmental, capable of judging the matching adaptability level of both. Moreover, the assessment mode can give a preliminary display on correlations between the product impression recognition and the poetry conception (impression and idea); it also can analyze the feasibility of the matching level. The product samples selected by the assessment mode in this study are judgmental. It can also explain the cognitive concept of product impression, but also interpret the invisible "poetry culture" elements form a non-figurative nature.

In the matching adaptability of product perception and poetry conception, having figurative elements i.e. "form and color" is easier to produce a high degree of matching adaptability.

In Table 3, in the matching of "iron-red crystalline glaze", ranking No. 1 "In sunrise flowers along the river are red like fire"; in the matching of "powder-green glaze”, ranking No. 1 "Beautiful Views of Fresh Green Mountain Peaks after Rain”. "Red” and "Green” are elements representing colors, and "Fire” and "Peak” are the elements representing forms. Therefore, it is known that the figurative elements, ie. "Form' and "Color" in the matching adaptability of product perception and poetry conception are easier to produce a high degree of matching adaptability.

It is recommended that when poetry culture with invisible abstraction is applied to the culturally creative design, it still require a figurative element to do the auxiliary operation so as to promote product perception and poetry conception for more intimate matching correlation.

\section{Analysis of Matching Adaptability of Product Perception and Poetry Conception and Comparative Analysis of Poetry Conception Preference (Test B and Test C)}

For the matching adaptability level of product perception and poetry conception, no personal preference on poetry conception would put into consideration.

Six products corresponding to the "Analysis of poetry conception preference", shown in Table 2. Among 18 in total ranked top three of the matching adaptability level of product perception and poetry conception, There were only 3 poems listed, respectively ranking No. 1 "When walking to a place where there is no water, Sit and watch clouds in the sky"; ranking No. 2 "Watch the bright moon through light clouds; see the green 
mountains through light fog”; ranking No. 5 "Beautiful Views of Fresh Green Mountain Peaks after Rain”. In poetry conception preference, ranking No. 3 "Every cloud has a silver lining" and the ranking No. 4 "but afraid of those large mansions; too high to keep out the cold" did not appear in the ranking items of the matching adaptability level of product perception and poetry conception.

Three items in ranking of other poetry conception preference appeared only once in the item of the matching adaptability level of product perception and poetry conception. It showed that the testees' poetry conception preference did not affect the level of product perception and poetry conception; for the matching adaptability level of product perception and poetry conception, no personal preference on poetry conception would put into consideration. In order to understand how to name the product design poetry, it is recommended to consider seeking the meaning of the theme in line with the poetry, rather than using personal preference on poetry conception. This study also showed a determination of the content filtering poetry. This study also showed the selected product samples are judgmental.

The matching adaptability of poetry conception preference and poetry conception are not lowly repeated

In poetry conception preference, ranking No. 1 "When walking to a place where there is no water, Sit and watch clouds in the sky"; It accounts for only (1/18) among the 18 items of the matching of poetry conception, and no high repeatability. It showed this assessment study has produced correlations and study findings of analysis of the matching between the product cognition and poetry conception. Based on this, we can apply the assessment mode in this study to carry out a preliminary exploration in the relevant factors, functions and purposes of "product cognition and poetry conception".

\section{Poetry Conception Preference (Test C)}

In the product design, when the poetry is to be named, the category "Fantasy" (Emotion) should be put into the top consideration.

The ranking of poetry conception preference is shown in Table 2. In the top five, ranking No. 2 "Watch the bright moon through light clouds; see the green mountains through light fog”; ranking No. 5 "Beautiful Views of Fresh Green Mountain Peaks after Rain”, which are in the category of meaning (simile analogy). The ranking No. 1 "When walking to a place where there is no water, Sit and watch clouds in the sky"(3.15); ranking No. 3 "Every cloud has a silver lining" and ranking No. 4 "but afraid of those large mansions; too high to keep out the cold", which are in the category of "Fantasy" (emotions, metaphor metonymy). In the poetry conception preference, it shows that the category "Fantasy" (emotions) serves as a priority selection. Based on this, we can realize that when poetry is to be named in the product design, it is preferable to put the poetry content of category "Fantasy" (emotions) into the top consideration.

\section{General Comparison Analysis}

After conducting relevant qualitative \& quantitative analyses, the preliminary findings show that the category "Fantasy" (emotion) expresses more subjective abstract, not likely to have highly connected matching, if deep thought and proper application of the "Fantasy" (emotion) would be given, a more appropriate convey of poetry is applied to the product design, it can then increase the cultural connotations of the product design. To have an effective and a good use, give names in the poetry conception preference, such as the preference No. 1 "When walking to a place where there is no water, Sit and watch clouds in the sky". To have this poetry for the design with a creative thinking, and go on in the design direction of poetry converting, it will enhance the correlations of the poetry artistic perception preferences and product perception . Furthermore, it can strengthen 
the meaning and value in this study of the application of "poetry culture" in the culturally creative design. In addition, six observing assessment indicators show that categories "meaning" "body" of poetry are easier to do match operations in the selection of the poetry conception of matching product impression. It is known that the "six observing" principle and designed converting dimensions still have considerations for the degree of difficulties and priorities. Operators should start the highly adaptable items step by step.

\section{Conclusion}

Li, Y.L. (2007) stated, the time is inexhaustible and the universe is infinite. While the human's thought goes on from one generation to another generations. As their visions travel miles after miles, poets will continue reaching eternal thoughts in space and time. Poetry is representative of endless life in performance. In the ancient and modern dialogue through time and space, the creative design applications of poetry culture will enable Chinese classical poetry to send out new majesty and grandeur of the times. This study selected the idea of David Hume's empirical theory, and then applied to six observing principle of Chinese "wen xin dio long" as assessment indicator. After questionnaires implementing three items, i.e. the matching adaptability of product color preference, product perception and poetry conception, and poetry conception, followed up to conduct qualitative and quantitative analyses of cross-comparison of related items. By doing so, the preliminary summary between product perception and poetry conception, and assessment references of concrete operation can be made. Furthermore, to understand how to promote the matching adaptability of product impression and poetry conception, and assessment indicators in views of the difficulty degree of poetry implications conversion, enable "poetry culture" to appropriately and concretely convey the Cognitive impression of creative product design.

Although in the study process, from the forming of theoretical concepts, the setting of assessment indicators, and the screening and classification of poetry meaning, they are all affected by many subjective factors. Compared to quantitative method of general science, it cannot give a precise interpretation and expression; however, undoubtedly it is a genuine value that promotes and "reproduces" literature and art. Consequently, the poetry and product perception are produced for the use of preliminary assessment and studies. In a hope that innovative assessment idea and direction can be submitted to help recognize the external form of product design and poetry connotation can be appropriately matched and adapted. And then the inheritance and inspiration of poetry culturally creative design can be effectively extended. Moreover, the aroma of ancient and traditional culture can be gloried and magnified.

\section{References}

Cassirer, E. (1979). Symbol, myth, and culture. London: Yale University Press.

Chu, K.C. (1988) ). Literary psychology . Taipei: Jinfeng.

Chou, C.F. Annotation (1993). wen xin diao long. Taipei: Chingshui.

Langer, S. K. (1953). Feeling and form: A theory of art developed from philosophy in a new key. NY: Charles Scribner's Sons.

Lin, R.T. (2010). Perceptual field and quality product from Creativity Life Industry - The One South Park case study. Department of Arts Process Design, School of Design of National Taiwan University, NSC Project No.: NSC-98-2410-H-144-009 \& 010.

Rapaille, C. (2006). The culture code. New York: Broadway Books.

Worringer, W. (2007). Abstraction and empathy: A contribution to the psychology of style. Chicago: Ivan R. Dee.

Wang, M.O. ( 2010 ) . Literary aesthetics, Taipei: Lijen.

Yu, C.Y. (2006). Arts Creationism. Taipei: World Vision.

Yu, Y. T. (2010). Wen Xin Diao Long literary philosophy discussion. Taipei: Student.

Ye, Moil., Lin, B.H., Chu, C.H.( 2011) "Culturally creative design application of poetry form conversion", "Design Journal", 16 (4), (pp. 91-106). 